\title{
QoS Aware Power and Hop Count Constraints Routing Protocol with Mobility Prediction for MANET Using SHORT
}

\author{
Senthilkumar Maruthamuthu ${ }^{1}$, Somasundaram Sankaralingam ${ }^{2}$ \\ ${ }^{1}$ Department of Computer Technology and Applications, Coimbatore Institute of Technology, Coimbatore, India \\ ${ }^{2}$ Department of Mathematics, Coimbatore Institute of Technology, Coimbatore, India \\ E-mail:msenthilkumar.cta.cit@gmail.com,somasudaram@cit.edu.in \\ Received November 16, 2010; revised January 15, 2011; accepted January 17, 2011
}

\begin{abstract}
A mobile ad hoc network (MANET) is composed of mobile nodes, which do not have any fixed wired communication infrastructure. This paper proposes a protocol called "Delay, Jitter, Bandwidth, Cost, Power and Hop count Constraints Routing Protocol with Mobility Prediction for Mobile Ad hoc Network using Self Healing and Optimizing Routing Technique (QPHMP-SHORT)". It is a multiple constraints routing protocol with self healing technique for route discovery to select a best routing path among multiple paths between a source and a destination as to increase packet delivery ratio, reliability and efficiency of mobile communication. QPHMP-SHORT considers the cost incurred in channel acquisition and the incremental cost proportional to the size of the packet. It collects the residual battery power of each node for each path; selects multiple paths, which have nodes with good battery power for transmission to satisfy the power constraint. QPHMP-SHORT uses Self-Healing and Optimizing Routing Technique (SHORT) to select a shortest best path among multiple selected paths by applying hops count constraint. It also uses the mobility prediction formula to find the stability of a link between two nodes.
\end{abstract}

Keywords: QoS, Power Consumption, Hop Count, Routing, Mobility Prediction, MANET

\section{Introduction}

Mobile ad hoc networks (MANETs) do not have any fixed wired communication infrastructure. They can be deployed in the applications such as military battlefields, emergency search, rescue sites, classrooms and conventions, where participants share information dynamically using their mobile devices [1]. The issues addressed in MANET are routing, mobility management, security, reliability and power consumption [2].

Quality of Service (QoS) in MANET is defined as the collective effect of service performance, which determines the degree of satisfaction of a user of the service [3]. The QoS constraints are classified as: Time constraints - (Delay, jitter); Space constraints - (System buffer); Frequency constraints - (Network/system bandwidth); and Reliability constraints - (Error rate). QoS is needed in MANET because different applications have different service requirements; for example, VoIP considers issues like delay, jitter and minimum bandwidth [2]. Some of the QoS models applicable for MANET are Integrated services (IntServ), Differentiated Services (DiffServ), Flexible QoS Model for MANET (FQMM), Complete and Efficient QoS Model for MANETs (CEQMM). The IntServ model is a stateful model. It maintains per flow reservation state at QoS network entities. It gives guaranteed bandwidth for flows.

The optimal allocation of bandwidth for nodes periodically to use shared or multiple wireless links is the NP complete problem. Due to huge resource consumption and computation power limitation, this model is not suitable for MANET [4]. The DiffServ model is a lightweight stateless model. There is no reservation of resources. The QoS can be achieved through the mechanisms namely Admission Control, Policy Manager, Traffic Classes and Queuing Schedulers. Intermediate nodes give higher priority to real-time traffic than bulk traffic. There is no absolute bandwidth guarantee for flows. It relies on the type of the traffic [5].

FQMM is a hybrid model where traffic conditioning 
scheme is used at ingress router. In FQMM, the highest priority traffic is governed by IntServ model, and DiffServ governs remaining data traffic [6]. CEQMM is also a hybrid model which combines IntServ and DiffServ models. The highest priority flows can be determined based on multiple metrics using IntServ. The remaining flows can be handled using DiffServ model. Due to control overhead, nodes consume more battery power. It specifies the QoS Optimized Link State Routing (QOLSR) protocol [7].

In-band signaling system for supporting QoS in MANET (INSIGNIA) is a QoS signaling protocol. It uses soft state method and manages each flow individually. It piggybacks resource reservations onto data packets, which can be modified by intermediate nodes to inform the communication endpoint nodes in case of lack of resources due to topological change. In INSIGNIA, the resource is allocated to a particular flow, if available. Otherwise, best effort service is performed [8]. Service Differentiation in Stateless Wireless Ad hoc networks (SWAN) is a stateless QoS model. It differentiates realtime and best effort packets and shapes only the besteffort packets using a leaky bucket traffic shaper algorithm. The per-flow reservation state is not maintained in intermediate nodes [9].

Some of the QoS routing algorithms for MANET are Core Extraction Distributed Ad hoc Routing (CEDAR), Ticket-Based Probing (TBP) and QoS-AODV (QAODV). CEDAR uses clustered network architecture and selects the core dynamically. Core node gets the details of high bandwidth and stable links. Low bandwidth and low stable links are used within each domain of a cluster. All cores are initially connected. Partial QoS paths are identified and concatenated at last. It suffers from control overhead and bottleneck at core [10].

The routing protocols in MANET can be categorized as proactive and reactive [11]. Proactive protocols are table driven which function on the basis of predefined tables constructed before communication takes place. Frequently tables are updated. Periodical exchanging of table information among nodes in dense area will create communication overhead. If more nodes are in mobility, it requires more updates in tables, which increases communication overhead. Reactive protocols are used when the source has data to send. These are suitable for high mobility. Both the protocols are used for route discovery and route maintenance between a source and a destination. In proactive routing, route discovery is easy but route maintenance is hard. In reactive routing, route discovery is hard but route maintenance is easy.

The Section 2 describes the literature review of QoS routing protocols. In Section 3, the network model of the new protocol has been explained. In Section 4, mobility prediction mechanism is explained. In Section 5, a new protocol QPHMP-SHORT with path discovery and path maintenance procedures is explained with an example. The Section 6 gives the simulation set up and the performance of QPHMP-SHORT. Finally Section 7 gives the conclusion and future scope of this research work.

\section{Literature Review}

QAODV is based on reactive routing. In QAODV, the source node specifies the QoS parameters in the RREQ packet. Every intermediate node checks whether or not it can support that QoS [12]. TBP is a multi-path QoS routing scheme. In TBP, the source node sends $\mathrm{N}$ number of tickets to find $\mathrm{N}$ paths. It has three types. In type I, resource reservation is done on more than one path and each packet is routed along each path. In Type II, resource reservations are done along multiple paths, but one path is used as a primary path. In type III, even though multiple paths exist, resources are reserved only on the primary path. There is no clear-cut heuristics for computing tickets. Resource Reservation for one flow denies the availability of that resource for other flows [13]. There can be multiple paths between a source and a destination. The links associated in each and every path between a source and a destination, have the QoS constraints based on the QoS parameters such as delay, jitter, bandwidth and cost. A path can be chosen as an optimal path if it satisfies all the above said constraints [11].

The protocol multiple QoS constraints routing protocol with mobile predicting (MQRPMP) discusses the QoS routing problem with multiple QoS constraints, the delay, delay jitter, bandwidth and cost metrics. It attempts to reduce the overhead for reconstructing a routing path with multiple QoS constraints by mobile predicting. It has better Packet Delivery Ratio (PDR) than TBP. The cost of communication overhead is also less than TBP [11]. The mobile nodes suffer from battery consumption in MANET. But MQRPMP does not consider power constraint, the incremental cost proportional to the size of the network layer packet and hop constraint to select a path. The Optimized Polymorphic Hybrid Multicast Routing (OPHMR) protocol [14] for MANET is based on the principle of adaptability and multi behavioral modes of operations.

It is able to change the behavior of nodes in different situations in order to improve certain metrics like maximizing battery life, reducing communication delays, improving deliverability, etc. The incremental cost proportional to the size of the network layer packet can be considered as a node constraint along with the cost incurred in channel acquisition [14]. The power consumption of 
nodes is also a known key constraint, which affects the performance of MANET [14]. It can be treated as a node constraint. By collecting the residual battery power of each and every node in a path; we can find a path, which has nodes with good battery power for transmission. But OPHMR does not address the impact of link constraints namely jitter and bandwidth.

The Temporarily Ordered Routing Algorithm with Self-Healing and Optimizing Routing Technique (TORASHORT) described in [15] shows better performance than Temporarily Ordered Routing Algorithm (TORA). The Self-Healing and Optimizing Routing Technique (SHORT) is a mechanism that optimizes the route length to reduce delay and increase throughput of communication [16]. A path with minimum hop counts can be selected as a shortest path using SHORT. But TORASHORT does not consider the link constraints namely jitter, bandwidth and cost.

The proposed protocol is a source based multi constraint reactive protocol with mobility prediction for finding optimal path in MANET. In which, if a path satisfies link and node constraints namely delay, jitter, bandwidth, with highest Link Expiry Time (LET), minimum hops, and good battery power whose cost is minimal can be selected as an optimal path.

\section{Network Model}

The network model in MANET can be denoted by $\mathrm{G}=$ $\{\mathrm{V}, \mathrm{E}\}$ where $\mathrm{V}$ is the set of interconnected nodes and $\mathrm{E}$ is the set of full-duplex directed wireless communication links. The network model considers the existence of multiple links between any two nodes and each link considers the QoS parameters namely Delay (D), Jitter (J) and Bandwidth (B). The links in the routing paths for the model should satisfy the QoS constraints. The Cost function $(\mathrm{C})$ can be modeled as a linear function as shown below where $\mathrm{b}$ is the fixed cost of channel acquisition and $\mathrm{m}$ is the incremental cost proportional to the size of network layer packet to be transmitted between the nodes $i$ and $j$. It can be the transmission cost of the packet.

The model also considers the Energy Level (EL) of each node $\left(V_{i}\right)$ on each path, which meets a power constraint $\left(\mathrm{P}_{\mathrm{c}}\right)$ for mobile communication. The EL of each node is the residual battery backup, which is collected and summed up for each routing path as to select the best path. Among the existence of multiple paths $\left(\mathrm{P}_{1}, \mathrm{P}_{2}\right.$, $\mathrm{P}_{3} \cdots$ and $\mathrm{P}_{n}$ ) between a source and a destination, a path $P_{k}$ is selected which satisfies the above said constraints. If $\mathrm{P}_{\mathrm{k}}$ represents multiple paths then the SHORT procedure is applied to find an optimum path with least hop count $(\mathrm{H})$ and highest LET where $\sum D_{i j}$ and $\sum J_{i j}$ are less as well as $\sum E L\left(V_{i}\right)$ is highest. During the selection, if a path has lowest LET with least hop count, it is rejected since it is not a stable path. If the path has highest $\sum E L\left(V_{i}\right)$ and highest hop count is also rejected since it contains more nodes where the accumulation of residual battery power of all the nodes is normally high.

So the problem of multiple QoS constraints with power awareness and optimized hop count can be defined as follows:

Select $\mathrm{P}_{\mathrm{k}}$ whose LET is largest among $\left(\mathrm{P}_{1}, \mathrm{P}_{2}, \mathrm{P}_{3} \cdots\right.$, $\left.P_{n}\right)$.

$$
\begin{aligned}
\text { Where } & \sum D_{i j} \leq D_{c} \\
& \sum J_{i j} \leq J_{c} \\
& B_{i j} \geq B_{c} \\
& C_{i j}=\mathrm{m} * \text { size }+b \\
& \operatorname{EL~}\left(V_{i}\right) \geq P_{c} \\
& \operatorname{MIN}\left(\sum H\right)
\end{aligned}
$$

Such that $\left(\sum D_{i j}\right.$ and $\left.\sum J_{i j}\right)<$ other paths

With MAX (LET)

$\operatorname{MIN}\left(\sum C_{i j}\right)$

$\operatorname{MAX}\left(\sum E L\left(V_{i}\right)\right)$

\section{Mobility Prediction Mechanism}

This section describes the mobility prediction formula for finding LET. In MANET, the reliability of a path is affected due to frequent changes in network topology. The reliability of a path depends on the stability or availability of each link of this path. It supposes a free space propagation model [17], where the received signal strength solely depends on its distance to the transmitter. Here the node-moving pattern is random waypoint. We also assume that all nodes in the network have their clock synchronized [e.g., by using the NTP (Network Time Protocol) or the GPS clock itself] [18]. Therefore, using the motion parameters such as speed, direction, and the communication distance of two neighbors, the duration of time can be determined in order to estimate that two nodes remain connected or not. The LET between any two nodes is calculated at each node using the well-known mobility prediction formula. Assume that two nodes $\mathrm{i}$ and $\mathrm{j}$ are within the transmission range $\mathrm{r}$ of each other. Let $\left(x_{i}, y_{i}\right)$ be the coordinate of mobile host $i$ and $\left(x_{j}, y_{j}\right)$ be that of mobile host $j$. Also let $v_{i}$ and $v_{j}$ be the speeds, and $\theta_{i}$ and $\theta_{j}$ be the moving directions of nodes $i$ and $j$ respectively. Then, the amount of time that they will stay connected-LET, is predicted by the formula given in the Equation (1).

$$
\text { LET }=\frac{-(a b+c d)+\sqrt{\left(\left(a^{2}+c^{2}\right) r^{2}-(a d-b c)^{2}\right)}}{\left(a^{2}+c^{2}\right)}
$$

where $a=v_{i} \cos \theta_{i}-v_{j} \cos \theta_{j}$,

$$
b=x_{i}-x_{j}
$$




$$
\begin{aligned}
& c=v_{i} \sin \theta_{i}-v_{j} \sin \theta_{j}, \text { and } \\
& d=y_{i}-y_{j}
\end{aligned}
$$

Note that when $v_{i}=v_{j}$ and $\theta_{i}=\theta_{j}$, LET is set to $\infty$ without applying the above equation.

\section{QoS Aware Power and Hop Constraints Routing Protocol with Mobility Prediction for MANET Using SHORT}

This section describes path discovery and path maintenance procedures for the proposed protocol. During path discovery, an optimal path is selected based on multiple QoS constraints and power constraint with mobility prediction formula. The two neighboring nodes use GPS information and LET is calculated [17]. The path with minimal cost, with good battery power and highest LET is considered as a stable and optimal path for transmission.

\subsection{Path Discovery}

When a source has data to send then it sends route request packet with following fields: Source-address, Destination-address, sequence number, position, speed, moving direction of mobile node, D, J, B, EL and C. The route discovery procedure for the proposed protocol is given as follows:

\section{Procedure for Source $(S)$ : \\ If Source $\mathrm{S}$ has no Paths to Destination D \\ Broadcast Route Request packet with $\mathrm{HC}$ as 0 \\ Execute Route Reply Handling Procedure \\ Endif}

Route Request Handling procedure:

If it is an intermediate node I

If the received Route Request packet is not duplicate

$$
\begin{aligned}
& \text { If ( } \left.B_{i j}>=B_{c}\right) \\
& \text { If Entry for (S,D) is found and the } \\
& \text { Difference in } \mathrm{HC}>2 \\
& \text { its hop comparison array } \\
& \text { Forward Route Request } \\
& \text { with } \mathrm{HC}=\mathrm{HC}+1 \\
& \text { with } \mathrm{HC}=\mathrm{HC}+1
\end{aligned}
$$$$
\text { Update Record (S, D, HC, PREV) in }
$$

$$
\text { End if }
$$

Else

$$
\text { End if }
$$

Discard Route Request

Else
Discard Route Request

End if

End if

If the node is D

If the received Route Request packet is not duplicate

Execute Route Reply Handling Procedure End if

End if

Route Reply Handling procedure:

If it is an intermediate node I or destination D

If the received Route Reply Packet is not

duplicate and EL $\left(V_{i}\right)>=P_{c}$

Update $\mathrm{EL}$ as $\mathrm{EL}=\mathrm{EL}+\mathrm{EL}$ (I) within

received Route Reply

Update Routing table

Forward new Route Reply to S

End if

Delete the corresponding Hop

Comparison entry in I

End if

If the node is $\mathrm{S}$

Receive the Route Reply packets

Collect the Paths to D

If the Collection is not NULL

For each Path $\mathrm{P}$

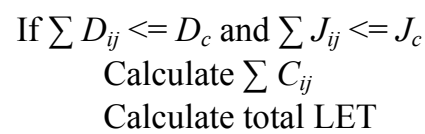

Else

Delete routing path

from the Collection

Endif

End for

Delete the corresponding

Hop Comparison entry in $\mathrm{S}$

Select $\mathrm{P}_{\mathrm{k}}$ with $\mathrm{HC}\left(\mathrm{P}_{\mathrm{k}}\right)$ is low and highest LET among multiple paths $\left(\mathrm{P}_{1}, \mathrm{P}_{2}, \mathrm{P}_{3} \ldots, \mathrm{P}_{\mathrm{n}}\right)$ given $\sum C_{i j}$ is minimum and $\sum E L\left(V_{i}\right)$ is maximum If $\mathrm{P}_{\mathrm{k}}$ has lowest LET with least hop count, reject $\mathrm{P}_{\mathrm{k}}$ Endif

If $\mathrm{P}_{\mathrm{k}}$ has highest $\sum E L\left(V_{i}\right)$ and highest hop count, Reject $\mathrm{P}_{\mathrm{k}}$ Endif

If LETs and/ or $\sum E L\left(V_{i}\right)$ of several $\mathrm{P}_{\mathrm{k}} \mathrm{s}$ are equal Select the least hop path Endif End if

End if 


\subsection{Path Maintenance}

Due to the frequent changes of network topology and restriction of network resources, the computed optimal route often gets invalid. When the link is cut off, the upstream node issues a route reconstruction packet to the source and the source starts the route discovery again. If the source receives route reply and route reconstruction packets at the same time, it deals with the route reconstruction packet.

Route maintenance procedure by the Intermediate node:

If the link is cut off with its neighbor

Construct and Send

Route Reconstruct packet to $\mathrm{S}$

End if

If the Route Reconstruct packet is received from its neighbor

Forward the Route Reconstruct packet to S

End if

Route maintenance procedure by the Source node:

If the Route Reconstruct packet is received from any I Broadcast Route Request packet

End if

\subsection{Example}

Figure 1 depicts a graph with nodes S, A, B, C, and D in (QPHMP-SHORT). The source $\mathrm{S}$ wants to transmit packets to destination D. Each link in Figure 1 is represented with the QoS metrics (D, J, B, C) such that C is expressed in terms of both node and link constraints. Let the links S-A, A-B, A-C, B-C, and C-D are having the QoS metrics $(2,4,50,9),(5,6,40,6),(7,10,37,10),(3,3,35,4)$, and $(4,3,39,7)$ respectively. Let the link thresholds $D_{c}=$ $15, J_{c}=30, B_{c}=35$ and node threshold $P_{c}=50$. Let the energy levels of the nodes S, A, B, C and D are 45, 40, 43, 43, and 35. In Figure 1, the node A is within the range of $\mathrm{S}$. The nodes $\mathrm{B}$ and $\mathrm{C}$ are within the range of node $\mathrm{A}$. The nodes $\mathrm{A}$ and $\mathrm{C}$ are within the range of node $\mathrm{B}$. The nodes $\mathrm{A}, \mathrm{B}$ and $\mathrm{D}$ are within the range of $\mathrm{C}$.

There exists two paths $\mathrm{P}_{1}(\mathrm{~S}, \mathrm{~A}, \mathrm{~B}, \mathrm{C}, \mathrm{D})$ and $\mathrm{P}_{2}(\mathrm{~S}, \mathrm{~A}$, $\mathrm{C}, \mathrm{D})$ from $\mathrm{S}$ to $\mathrm{D}$. These two paths are satisfying $D_{c}, J_{c}$, $B_{c}$ and $P_{c}$. The minimum cost of the two paths is equal. Both paths are having equal LET values. But $\mathrm{P}_{1}$ has good battery backup than $\mathrm{P}_{2}$. By applying the SHORT mechanism as explained below, Table $\mathbf{1}$ is created. From Table 1, it is identified that the path $\mathrm{P}_{2}(\mathrm{~S}, \mathrm{~A}, \mathrm{C}, \mathrm{D})$ has least hop count.

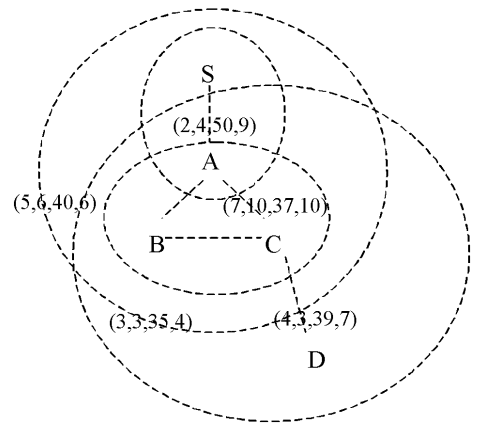

Figure 1. Nodes with QoS metrics and transmission range.

Table 1. The entry of the hop comparison array.

\begin{tabular}{|c|c|c|c|c|c|c|}
\hline & $\mathrm{S}$ & A & B & C & D & Shortcut \\
\hline (i) & $(0, S)$ & $(0, S)$ & - & - & - & - \\
\hline (ii) & $(0, S)$ & $(0, S)$ & $(1, \mathrm{~A})$ & $(1, A)$ & - & - \\
\hline (iii) & $(0, S)$ & $(0, S)$ & $(1, \mathrm{~A})$ & $(2, B)$ & - & - \\
\hline (iv) & $(0, S)$ & $(3, C)$ & $(1, \mathrm{~A})$ & $(2, B)$ & $(3, C)$ & Found at $\mathrm{A}$ \\
\hline (v) & $(0, S)$ & $(0, S)$ & - & $(1, A)$ & $(2, \mathrm{C})$ & \\
\hline
\end{tabular}

The path $P_{2}$ is selected as the best route than $P_{1}$ because the total end-to-end delay $\sum \mathrm{D}_{\mathrm{ij}}$ and end-to-end jitter $\sum \mathrm{J}_{\mathrm{ij}}$ is very low due to least hop count as well as path maintenance is so easy when number of links are reduced.

i) S needs to forward a packet to A. An entry is initialized in S's hop comparison array as (S, D, 0, S). It broadcasts the packet while marking $\mathrm{HC}$ as 0 . Node $\mathrm{A}$ is within the transmission range of $\mathrm{S}$ and receives the broadcast. So it records $(\mathrm{S}, \mathrm{D}, 0, \mathrm{~S})$ in its hop comparison array.

ii) Node A broadcasts the packet with a HC of 1 destined for D. Nodes B and C also receive the packet as they are in the transmission range of $\mathrm{A}$. $\mathrm{B}$ and $\mathrm{C}$ record $(\mathrm{S}, \mathrm{D}, 1, \mathrm{~A})$ in their hop comparison array.

iii) Node B broadcasts the packet with a $\mathrm{HC}$ of 2 . Nodes $\mathrm{A}$ and $\mathrm{C}$ have an entry corresponding to $(\mathrm{S}, \mathrm{D})$. Since the difference in HCs not more than 2, nothing is updated.

iv) While forwarding the packet to D, C broadcasts the packets with a $\mathrm{HC}$ of 3 . Nodes $\mathrm{A}, \mathrm{B}$, and $\mathrm{D}$ are in the transmission range of $\mathrm{C}$. Node $\mathrm{D}$ consumes the packet as it is the destination node. B compares the $\mathrm{HC}$ with its stored entry. As the difference in $\mathrm{HC}$ is not more than 2, nothing is updated at B. At A, the HC difference is 3 .

So, node A then updates its own routing table to point to $\mathrm{C}$ as the next hop node for destination $\mathrm{D}$.

v) The corresponding entry of the hop comparison array at $\mathrm{S}, \mathrm{A}$, and $\mathrm{C}$ is deleted. Thus, path $\mathrm{A}-\mathrm{B}-\mathrm{C}$ is shortened to A-C. The final path is shown in Figure 2. This 
shortcut path formation is termed as $(2,1)$ reduction.

The Table 2 shows the comparison of the paths $\mathrm{P}_{1}$ and $\mathrm{P}_{2}$ in terms of cost, energy level, LET and hops count. The cost and LET values are equal for $P_{1}$ and $P_{2}$. The path $\mathrm{P}_{2}$ has least hop count and less energy level than $\mathrm{P}_{1}$. The path $\mathrm{P}_{2}$ can be selected as an optimal path since $\sum D_{i j}$ and $\sum J_{i j}$ are less in $\mathrm{P}_{2}$ than $\mathrm{P}_{1}$. As well as, link maintenance is easy in $\mathrm{P}_{2}$ than $\mathrm{P}_{1}$.

\section{Simulation}

The protocol QPHMP-SHORT is simulated in ns2 [19]. The simulation parameters are shown in the following Table 3. The parameters used for evaluating QPHMPSHORT are cost of control overhead and success rate of data transmission. It is compared with MQRPMP and TBR in Figure 2.

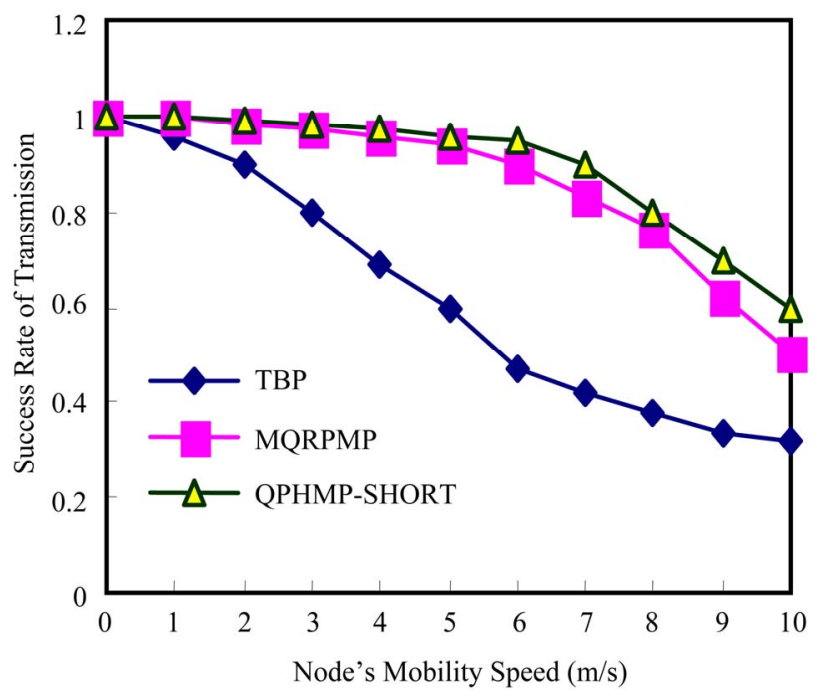

Figure 2. Success rate of data transmission vs. Node's mobility speed.

Table 2. Selection of optimal path.

\begin{tabular}{ccc}
\hline Metrics & Path $\mathrm{P}_{1}$ & Path $\mathrm{P}_{2}$ \\
\cline { 2 - 3 } & $\mathrm{P}_{1}(\mathrm{~S}, \mathrm{~A}, \mathrm{~B}, \mathrm{C}, \mathrm{D})$ & $\mathrm{P}_{2}(\mathrm{~S}, \mathrm{~A}, \mathrm{C}, \mathrm{D})$ \\
\hline$\sum D_{i j}$ & 14 & 13 \\
$\sum J_{i j}$ & 16 & 17 \\
$\sum C_{i j}$ & 26 & 26 \\
$\sum E L\left(V_{i}\right)$ & 206 & 163 \\
LET & 4 & 4 \\
Hops & 4 & 3 \\
\hline
\end{tabular}

Table 3. Simulation scenario.

\begin{tabular}{lc}
\hline \multicolumn{1}{c}{ Simulation Parameters } & Values given \\
\hline MAC Layer & IEEE 802.11 \\
Simulation Area & $500 \mathrm{~m} \times 500 \mathrm{~m}$ \\
Simulation Time & $500 \mathrm{~s}$ \\
Node Mobility Speed & $20-100 \mathrm{~m} / \mathrm{s}$ \\
Node Moving Pattern & Random Way Point \\
Traffic Type & CBR \\
Packet Size & 1024 bytes \\
The average end to end delay & $0.2 \mathrm{~s}$ \\
Transmission range & $250 \mathrm{~m}$ \\
\hline
\end{tabular}

The success rate of data transmission is compared with the node's mobility speed in Figure 2 to show the performance of QPHMP-SHORT over MQRPMP and TBR. When the node's mobility speed is $3 \mathrm{~m} / \mathrm{s}$ then the success rate of data transmission of MQRPMP and QPHMPHORT reaches the value 0.98 which is higher than the TBR value 0.8 . But while increasing the node's mobility speed above $3 \mathrm{~m} / \mathrm{s}$, the performance of MQRPMP, and TBR is drastically going down.

But among them, in QPHMP-SHORT the success rate of data transmission is still higher than the others and reaches 0.6 when node's mobility speed is 10 , since it uses the mobility prediction mechanism to keep stable links and path with good battery backup of nodes for effective transmission.

Figure 3 shows the comparison of number of nodes with cost of control overhead incurred during transmission for QPHMP-SHORT, MQRPMP, and TBR. When increasing the number of nodes in communication, the cost of transmitting control packets also increases but it is less in QPHMP-SHORT and MQRPMP than TBR.

Since QPHMP-SHORT collects the residual battery backup of each node along the path during route reply as exactly in MQRPMP, there is no performance difference between QPHMP-SHORT and MQRPMP which is shown in Figure 3.

\section{Conclusions}

This paper discusses the new protocol QPHMP-SHORT with multiple QoS constraints based on the QoS parameters namely delay, jitter, bandwidth, and cost metrics between source and destination. It also considers power constraint for nodes for efficient packet transmission. It uses mobility prediction formula for LET calculation to select a stable path with minimal cost. It also specifies the network model for QPHMP-SHORT. The QPHMPSHORT provides a quick response to changes in the 


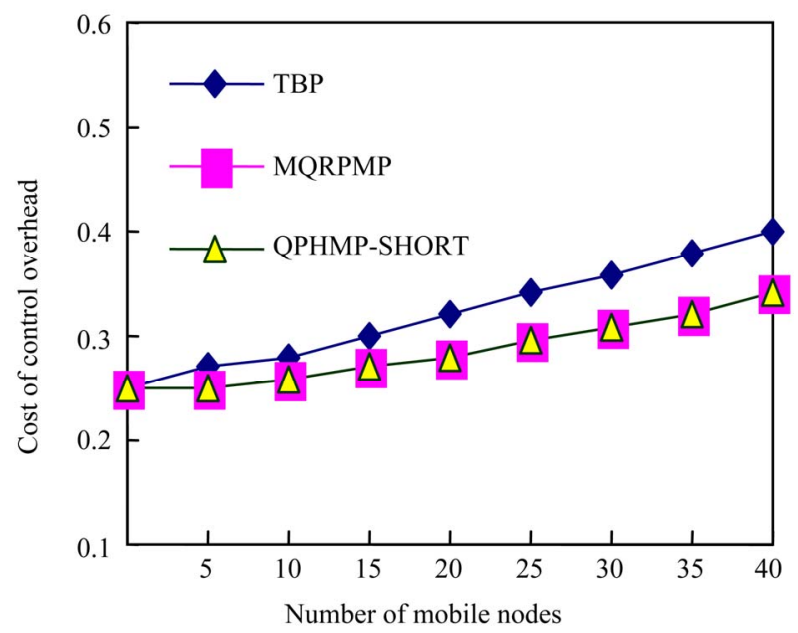

Figure 3. Cost of control overhead vs Number of mobile nodes.

network, minimizes the waste of network resources, produces significant improvement in data transmission rate, and reduces control overhead for reconstructing a routing path. It can be improved as a reliable and secure protocol for MANET. It can also be extended to be a hybrid multicast communication protocol. In future, the number of route request packets in route discovery can be reduced so as to increase effectiveness of the throughput of the communication.

\section{References}

[1] J. H. Luo, L. Xue and D. X. Ye, "Research on Multicast Routing Protocols for Mobile Ad-Hoc Networks," Computer Networks, Vol. 52, No. 5, April 2008, pp. 988-997.

[2] S. Ahmed and A. K. Ramani, "Exploring the Requirements for QoS in Mobile Ad Hoc Networks," Journal of Information \& Communication Technology, Vol. 1, No. 2, 2007, pp. 1-9.

[3] J. Jung, "Quality of Service in Telecommunications Part I: Proposition of a QoS Framework and Its Application to B-ISDN," IEEE Communications Magazine, Vol. 34, No. 8, August 1996, pp. 108- 111. doi:10.1109/35.533928

[4] IETF Network Working Group, "Integrated Services in the Internet Architecture: An Overview," IETF RFC: 1633, June 1994. http://www.ietf.org/rfe/rfc1633.txt

[5] S. Blake, "An architecture for Differentiated Services," IETF RFC2475, December 1998.

[6] H. Xiao, W. K. G. Seah, A. Lo and K. C. Chua, "A Flexible Quality of Service Model for Mobile Ad-Hoc Networks," Proceedings of IEEE Vehicular Technology Conference, Tokyo, 15-18 May 2000, Vol. 1, pp. 445-449.

[7] H. Badis and K. A. Agha, "CEQMM: A Complete and Efficient Quality of Service Model for MANETs," Proceedings of the 3rd ACM International Workshop on Per- formance Evaluation of Wireless Ad Hoc, Sensor and Ubiquitous Networks, Malaga, 2-6 October 2006, pp. 2532.

[8] B. Lee, G. S. Ahn, X. Zhang and A. T. Campbell, "INSIGNIA: An IP Based Quality of Service Framework for Mobile Ad Hoc Networks," Journal of Parallel and Distributed Computing (Special Issue on Wireless and Mobile Computing and Communications), Vol. 60, No. 4, 2000, pp. 374-406.

[9] A. S. Ahn, A. T. Campbell, A. Veres and L. Sun, "Supporting Service Differentiation for Real-Time and Best Effort Traffic in Stateless Wireless Ad Hoc Networks (SWAN)," IEEE Transactions on Mobile Computing, Vol. 1, No. 3, 2002, pp. 197-207.

[10] P. Sinha, R. Sivakumar and V. Bharghavan, "CEDAR: A Core-Extraction Distributed Ad Hoc Routing Algorithm," IEEE Journal on Selected Areas in Communications, Vol. 17, No. 8, 1999, pp. 1454-1465. doi:10.1109/49.779926

[11] J. Lian, L. Li and X. Zhu, "A Multiple QoS Constraints Routing Protocol Based on Mobile Predicting in Ad Hoc Network," Proceedings of IEEE International Conference on Wireless Communications, Networking and Mobile Computing, Shanghai, 21-25 September 2007, pp. 1608-1611.

[12] E. M. Royer, C. E. Perkins and S. Das, "Quality of Service for Ad Hoc on Demand Distance Vector (QAODV) Routing," IETF MANET, Internet Draft, draft-ietf-manetaodvqos-00, July 2000. http://www.cs.ucsb.edu/ ebelding/txt/qos.ps

[13] S. Chen and K. Nahrstedt, "Distributed Quality-of-Service in Ad Hoc Networks," IEEE Journal on Selected Areas in Communications, Vol. 17, No. 8, 1999, pp. 1488-1505. doi:10.1109/49.780354

[14] A. B. Mnaouer, L. Chen, C. H. Foh and J. W. Tantra, “An Optimized Polymorphic Hybrid Multicast Routing Protocol for MANET," IEEE Transactions on Mobile Computing, Vol. 6, No. 5, May 2007, pp. 551-562. doi:10.1109/TMC.2007.1030

[15] R. Asokan, A. M. Natarajan and C. Venkatesh, "Qualityof-Service Routing Using Path and Power Aware Techniques in Mobile Ad Hoc Networks," Journal of Computer Systems, Networks, and Communications, Vol. 2008, pp. (160574)1-7.

[16] C. Gui and P. Mohapatra, "A Self-Healing and Optimizing Routing Technique for Ad Hoc Networks," Technical Report CSE-2002-23, Department of Computer Science, University of California, Davis, 2002.

[17] W. Su, S. J. Lee and M. Gerla, "Mobility Prediction in Wireless Networks," Proceedings of 21 st Century Military Communications Conference, Los Angeles, 22-25 October 2000, Vol. 1, pp. 491-495.

[18] E. D. Kaplan, "Understanding the GPS: Principles and Applications," Artech House, Boston, February 1996. http://www.navtechgps.com/ Downloads/1024.PDF

[19] K. Fall, "The NS Manual, the VINT Project, a Collaboration between Researchers at UC Berkeley, LBL, USC/ISI and Xerox PARC," 2001. http://www.isi.edu/nsnam/ns 